\title{
Parameter estimation to study the immediate impact of aortic cross-clamping using reduced order models
}
J. Ventre ${ }^{1 *}$
M. T. Politi 2,3
J. M. Fernández $z^{2,3}$
A.
R. Ghigo 4
J. Gaudric ${ }^{1,5}$
S. A. Wray ${ }^{6}$
J.-B.
Lagaert $^{7}$
R. Armentano ${ }^{8}$
C. Capurro ${ }^{2,3}$
J. M.

\section{Fullana $^{1}$ | P.-Y. Lagrée ${ }^{1}$}

${ }^{1}$ Sorbonne Université, CNRS, Institut Jean Le Rond d'Alembert, Paris, France.

${ }^{2}$ Universidad de Buenos Aires, Facultad de Medicina. Departamento de Ciencias

Fisiológicas, Laboratorio de Biomembranas, Buenos Aires, Argentina.

${ }^{3}$ CONICET-Universidad de Buenos Aires.

Instituto de Fisiología y Biofísica "Bernardo Houssay", Buenos Aires, Argentina.

${ }^{4}$ Institut de Mécanique des Fluides de

Toulouse (IMFT), Université de Toulouse,

CNRS, INPT, UPS, Toulouse, France.

${ }^{5}$ Service de Chirurgie Vasculaire, Hôpitaux

Universitaires La Pitié-Salpêtrière, Paris,

France.

${ }^{6}$ Instituto de Medicina Traslacional,

Trasplante y Bioingeniería, Universidad

Favaloro-CONICET, Buenos Aires,

Argentina.

${ }^{7}$ Université Paris Sud, Laboratoire de mathématiques d'Orsay, CNRS, France.

${ }^{8}$ Departamento de Ingeniería Biológica, Universidad de la República, Montevideo, Uruguay.

\section{Correspondence}

J. Ventre, Sorbonne Université, CNRS, Institut Jean Le Rond d'Alembert, UMR 7190, F-75005 Paris, France.

Email: jeanne.ventre@dalembert.upmc.fr
Aortic cross-clamping is a common strategy during vascular surgery, however, its instantaneous impact on hemodynamics is unknown. We, therefore, developed two numerical models to estimate the immediate impact of aortic clamping on the vascular properties. To assess the validity of the models, we recorded continuous invasive pressure signals during abdominal aneurysm repair surgery, immediately before and after clamping. The first model is a zero-dimensional (OD) three-element Windkessel model which we coupled to a gradient-based parameter estimation algorithm to identify patient-specific parameters such as vascular resistance and compliance. We found a $10 \%$ increase in the total resistance and a $20 \%$ decrease in the total compliance after clamping. The second model is a 9-artery network corresponding to an average human body in which we solved the one-dimensional (1D) blood flow equations. With a similar parameter estimation method and using the results from the OD model, we identified the resistance boundary conditions of the 1D network. Determining the patient-specific total resistance and the distribution of peripheral resistances through the parameter estimation process was sufficient for the 1D model to accurately reproduce the impact of clamping on the pressure waveform. Both models gave an accurate description of the pressure wave and had a high correlation $\left(R^{2}>0.95\right)$ with experimental blood pressure data.

\section{KEYWORDS}

Clamping, OD model, 1D model, inverse problem, parameter estimation

This article has been accepted for publication and undergone full peer review but has not been through the copyediting, typesetting, pagination and proofreading process, which may lead to differences between this version and the Version of Record. Please cite this article as doi: $10.1002 / \mathrm{cnm} .3261$ 


\section{INTRODUCTION}

Aortic aneurysm (AA), abnormal dilations of the aorta, are responsible for over 150,000 deaths each year [1]. As the aneurysm grows, vascular surgery becomes necessary to relieve wall tension and prevent AA rupture [2]. Open surgery is currently the gold standard for elective AA repair with suitable anatomy and requires aortic cross-clamping [2]. Clamping consists of an external compression of the artery to prevent blood from flowing downstream, thus providing a more stable operative field. This procedure produces perturbations in cardiovascular hemodynamics ([3], [4]) but the exact mechanisms involved are not completely clear.

There is clinical evidence that the duration of cross-clamping is associated with postoperative clinical outcomes, including mortality ([5], [6]). Indeed, many experimental studies in animals [7] and humans [8] have reported the hemodynamic changes caused by aortic clamping on gastrointestinal function [9], on cardiac output ([10], [11], [12]) and renal perfusion [13]. Nevertheless, only changes produced 5 to 60 minutes after aortic clamping have been described, whereas immediate changes remain unknown. Therefore, there is a gap in current knowledge as to which are the immediate changes after aortic clamping, and what is the effect of clamping on the hemodynamics and vascular properties. Though some mechanical modeling studies have investigated the local stress distribution [14] to that causes arterial tissue damage produced by traumatic surgical instruments [15], to our knowledge, aortic cross-clamping has not been investigated using macroscopic blood flow models. Numerical models of the arterial network can provide information on important vascular features including compliance and resistance, given easily-obtained routine hemodynamic information such as continuous arterial pressure signals. In this study, we propose to investigate the immediate impact of aortic clamping on the vascular properties using two macroscopic numerical models.

The numerical models for blood flow in arteries are based on fluid-structure interaction (FSI) methods where fluid dynamics and flexible wall movement equations are coupled. In these models, blood flow is governed by the three-dimensional (3D) Navier-Stokes equations, and the wall deformation by visco-elastic solid equations. This 3D approach is usually restricted to small regions of the systemic circulatory system ([16], [17]) and only in a few cases is applied to the entire circulation [18]. However, the computational and modeling costs are high, making the 3D FSI simulation inadequate for real-time medical application. In contrast, reduced order models, such as zero-dimensional (OD) and one-dimensional (1D) models, are cost-effective alternatives to 3D FSI models.

Zero-dimensional models, also called lumped parameter models, are the simplest approach to describe blood flow. They are derived by averaging blood flow equations over all spatial dimensions and can be interpreted as an electrical analogy where voltage is assimilated to pressure and current to flow rate. The universal model is the Windkessel model [19], introduced by Frank [20] and used to describe the compliant charge and discharge of the aorta. The Windkessel model has been extensively applied ([21], [22]) to describe the flow of blood in the heart ([23], [24], [25], [26]) and other organs [27], to create models of the cerebral arterial network [28], of the entire arterial network ([29], [30]) and to characterize the microcirculation at the outlet of 3D FSI models ([31], [32]) and 1D network models ([33], [34], [35]). The major drawback of OD models is that they are unable to take into account wave propagation and nonlinear flow effects.

To overcome the OD limitations, one-dimensional models were introduced as the next level of modeling. The 1D equations are obtained by averaging the long wavelength Navier-Stokes equations over the cross-section of the artery. Conversely to OD models, 1D models account for pulse wave propagation and reflection in the arterial network which are important phenomena for understanding cardiovascular hemodynamics [36]. This model was introduced by Euler in 1775 [37] and is widely used to model blood flow in large arteries ([23], [38], [39], [40], [26]) in both healthy and pathological settings in humans ([33], [41]) and animals [42].

Changes in vascular properties have been linked to the pathogenesis of arterial hypertension [43] and atherosclero- 
sis [2], and therefore are thought to have a major role in the worldwide burden of cardiovascular diseases. However, clinicians usually have access to only minimal, if any, information on the vascular properties of their patients. Our study presents experimental data of continuous invasive arterial pressure recorded during AA repair surgery, before and after clamping the abdominal aorta. In order to enhance the data acquired from routine monitoring studies, we introduced a parameter estimation strategy, based on the experimental measurements, to identify valuable information on vascular mechanics that is otherwise difficult to obtain. Parameter estimation can also allow following the evolution of a disease in a minimally invasive way. A common parameter estimation technique is the Kalman filter ([44], [45]) developed to estimate the elastic properties of arterial vessels and the values of terminal boundary condition coefficients [46]. Others are retropropagation [47], adjoint-based methods [48] or quasi-Newton methods [49], for instance.

In this study, we compare two numerical models (OD and 1D) and experimental blood pressure measurements in order to investigate the immediate impact of aortic cross-clamping. We will show that both the OD and 1D models provide helpful information for medical diagnosis, at systemic and arterial scale respectively.

The paper is organized as follows: in Section 2 we present the experimental data acquisition method, we describe both the OD Windkessel model and 1D model and introduce the optimization method to identify the optimal parameters. Section 3 shows the results obtained with the numerical models. We discuss these results in Section 4 and finally, in Section 5 , we summarize our work and give perspectives for improvement.

\section{2 | METHODS}

We introduce first the data acquisition method (Sec. 2.1). We then present the two numerical models (Sec. 2.2 and 2.3). Finally (Sec. 2.4), we explain the parameter estimation method.

\subsection{Experimental data acquisition}

As stated above, we recorded arterial pressure signals of a patient undergoing an abdominal aneurysm (AA) repair intervention that required aortic cross-clamping at the Hôpital Universitaire Pitié-Salpétrière in Paris, France. We obtained the continuous and invasive pressure data with a fluid-filled catheter from the right radial artery of the patient during the surgery. The use of invasive arterial pressure measurements during this type of surgery is part of the surgical protocol and, therefore, routine clinical practice. The recording of these pressure data does not modify the signal used for clinical monitoring nor presents additional risks for the patient during the surgery.

To carry out the measurements, we used a disposable pressure transducer (TruWave, Edwards Lifescience $R$ ). We recorded the pressure signals with an analog-digital converter with internal hardware filters (low pass frequency set at $20 \mathrm{kHz}$, high pass frequency set at $0.05 \mathrm{~Hz}, \mathrm{MP} 150$, BIOPAC Systems Inc.). We used the AcqKnowledge software to interpolate arterial pressure data at a frequency of $1000 \mathrm{~Hz}$.

We performed the measurements on an 84-year-old male patient with hypertension and no left ventricle dysfunction (i.e. left ventricle ejection fraction < $45 \%$ ). We recorded the pressure data in two different configurations: the pre-clamp configuration as prior to aortic clamping (Fig. 1(a)) and the post-clamp configuration during which the clamp was in place (Fig. 1(b)). For each configuration, we chose a stable set of beats manually through a 20 to 40 -second interval immediately before and after each clamp event. We averaged the signal by calculating the period of each beat, taking the median period and normalizing every beat to this median period so that $50 \%$ of the beats were shortened and $50 \%$ were lengthened by the normalization. In this study, we compared the invasive experimental measurements with the numerical models on this single mean beat averaged from the 20 to 40 -second sequence for each configuration. 
(a)

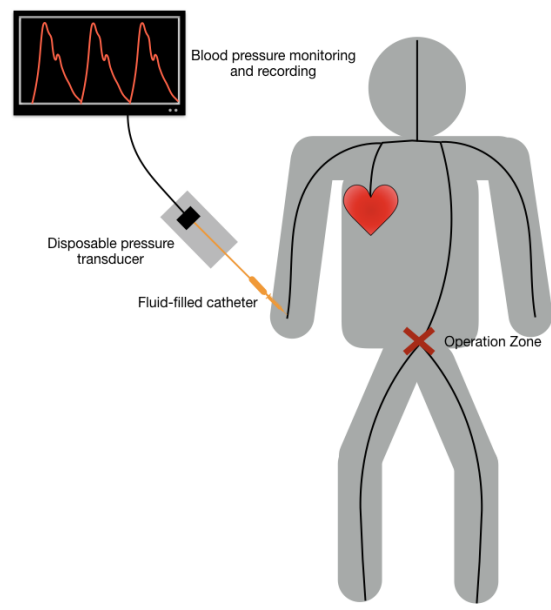

(b)

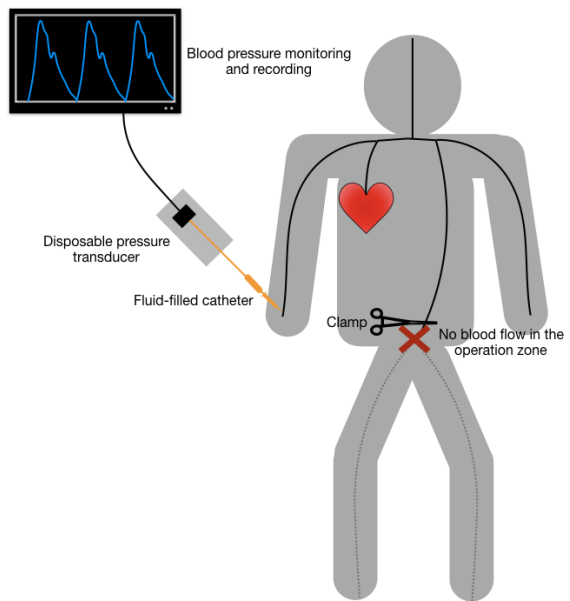

FIGURE 1 Schematic representation of the clamping procedure and data collection performed on a patient undergoing AA repair surgery. The fluid-filled catheter is positioned in the right radial artery, linked to a pressure transducer and monitor that records the blood pressure signal. We represent (a) the situation before clamping (pre-clamp) and (b) the abdominal clamping (post-clamp), both during AA repair surgery.

\section{$2.2 \mid$ Windkessel model}

\section{OD equation}

The Windkessel models are widely used in medical studies because, despite their simplicity and low computational cost, they provide valuable information on the vascular system. They were derived by averaging the blood flow equations over all spatial dimensions. The arterial network can then be interpreted as an electrical circuit constituted of resistors and capacitors. Resistors represent frictional forces due to viscosity while capacitors model the compliant effects of arteries. Windkessel models can therefore provide information on vascular resistances and compliances that are helpful for the medical diagnosis.

We propose a three-element Windkessel model composed by one capacitor $C$ and two resistors $R_{p}$ and $R_{d}$ corresponding to the proximal and distal resistances respectively (see Fig. 2(b)). The analytical representation of the Windkessel model in Fig. 2(b) is given by the following equation that links the pressure $p(t)$ to the flow rate $Q(t)$ ejected by the heart:

$$
\left(1+R_{d} C \frac{\mathrm{d} \cdot}{\mathrm{d} t}\right) p(t)=\left(\left(R_{p}+R_{d}\right)+R_{p} R_{d} C \frac{\mathrm{d} \cdot}{\mathrm{d} t}\right) Q(t)
$$

\section{Boundary conditions}

To minimise the number of model parameters, we chose to describe the flow rate $Q(t)$ ejected by the heart with a simple half sine signal. This heart model has proven reliable in previous works ([28], [40]), even though more complicated heart models can be found in the literature ([23], [24], [26]). The input flow rate follows 
(a)

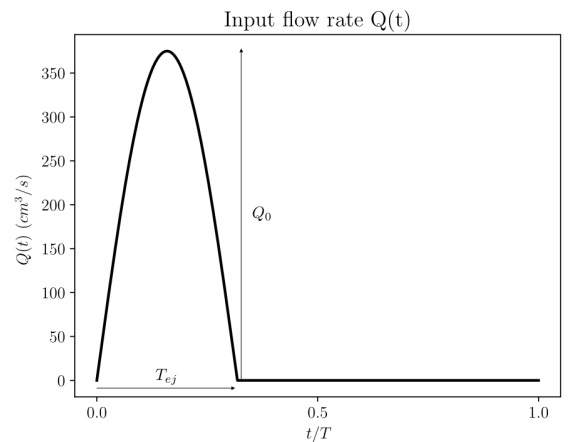

(b)

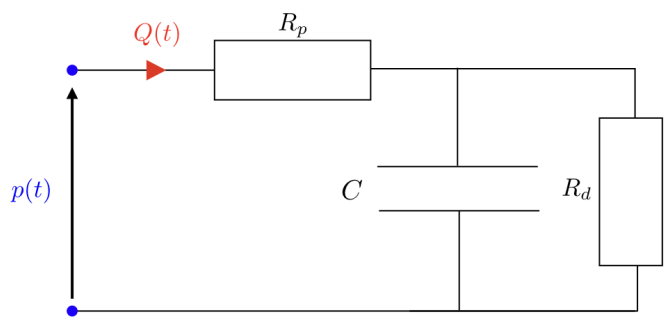

FIGURE 2 Windkessel OD model. (a) Inlet boundary condition of the OD and 1D models: flow rate $Q(t)$ modeling the heart. The amplitude is $Q_{0}=375 \mathrm{~cm}^{3} / \mathrm{s}$, the ejection time is $T_{e j}=31.8 \%$ of the heart period $T=1.15 \mathrm{~s}$. (b) Electrical representation of the three-element Windkessel model describing the entire systemic circulation. The pressure $p(t)$ is linked to the flow rate $Q(t)$, that models the heart through Eq. (1) where $R_{p}$ is the proximal resistance, $R_{d}$ is the distal resistance and $C$ is the compliance.

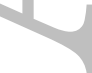

$$
Q(t)= \begin{cases}Q_{0} \sin \left(\frac{\pi t}{T_{e j} T}\right) & \text { if } 0<t<T_{e j} T, \\ 0 & \text { if } T_{e j} T \leq t<T,\end{cases}
$$

characterized by three parameters: the amplitude $Q_{0}$ and the systolic ejection time $T_{e j}$, during which blood is injected in the system, and the heart period $T$. The first two parameters $Q_{0}$ and $T_{e j}$ are unknown whereas the heart period is prescribed by the experimental data.

\section{Numerical resolution scheme}

To solve the equation (1) numerically, we used an explicit Euler time-integration scheme

$$
p^{n}+R_{d} C \frac{p^{n+1}-p^{n}}{\Delta t}=\left(R_{p}+R_{d}\right) Q^{n}+R_{p} R_{d} C \frac{Q^{n+1}-Q^{n}}{\Delta t}
$$

where $p^{n+1}$ is the unknown pressure at time $t^{n+1}$ and $Q^{n}, Q^{n+1}$ the known flow rates at times $t^{n}$ and $t^{n+1}$ respectively, imposed through the inlet boundary condition (2). The time step $\Delta t=t^{n+1}-t^{n}$ is of order $10^{-4} \mathrm{~s}$ and $\Delta t \ll R_{d} C \simeq 1 \mathrm{~s}$, the characteristic time of diastolic exponential decrease.

\subsection{One-dimensional model}

\section{D equations}

The 1D models have been used to describe blood flow in large arteries since, unlike OD models, they can model the spatial propagation of pulse waves, one of the most important phenomena when studying large artery hemodynamics ([38], [39], [50]). The 1D equations are obtained from the 3D Navier-Stokes equations assuming a few weak hypotheses. The details of the derivation can be found in [47], [50]. We assume that blood is homogeneous and behaves like a 
Newtonian fluid, and we consider arteries as straight axisymmetric tubes with viscoelastic walls. Finally, we average the long wavelength incompressible Navier-Stokes equations over the cross-section of the tube and obtain the following continuity and momentum equations:

$$
\left\{\begin{array}{l}
\frac{\partial A}{\partial t}+\frac{\partial Q}{\partial x}=0 \\
\frac{\partial Q}{\partial t}+\frac{\partial}{\partial x}\left(\frac{Q^{2}}{A}+\frac{K}{3 \rho} A^{\frac{3}{2}}\right)=-C_{f} \frac{Q}{A}+C_{\nu} \frac{\partial^{2} Q}{\partial x^{2}}
\end{array}\right.
$$

where $Q$ is the flow rate, $A$ the cross sectional area and $p$ the internal average pressure. The parameter $K=E \sqrt{\pi} h /\left(A_{0}\left(1-v^{2}\right)\right)$ characterizes the elastic behavior of the arterial wall and depends on the Young's modulus $E$, the wall thickness $h$, the Poisson coefficient $v$ and the reference arterial cross-section $A_{0}$. The blood density $\rho$ is equal to $1 \mathrm{~g} / \mathrm{cm}^{3}$. The friction coefficient $C_{f}$ is set to $22 \pi v$ [51] with $v=510^{-2} \mathrm{~cm}^{2} / \mathrm{s}$ the kinematic viscosity of blood. Finally, $C_{v}=\phi \sqrt{\pi} h /\left(2 \rho \sqrt{A_{0}}\left(1-v^{2}\right)\right)$ describes the visco-elastic behavior of the wall using a Kelvin-Voigt model, linearized around the reference cross-section $A_{0}$ ([35], [42]) and depends on $\phi$ the wall visco-elastic coefficient.

\section{Boundary conditions}

To model the systemic circulation in the pre-clamp configuration, we constructed a minimal network composed of nine main arteries [52] (see Fig. 3(a)) where we solved the 1D equations (4). At each bifurcation, we imposed the conservation of mass and the continuity of energy [53] without taking into account pressure losses at the junctions [54]. The boundary conditions are the following: at the inlet of the network (artery number 1 ) we imposed the same periodic flow input signal $Q(t)$ as the OD model (2) and we applied resistance boundary conditions $r_{i}$ at every terminal artery $i$ to take into account the peripheral circulation in the capillaries ([35], [55]). The resistance boundary conditions are not prescribed but estimated with the estimation process described in Section 2.4. The post-clamp configuration consists of removing all distal arteries starting from the end of the abdominal aorta (artery 7). In practice, we applied a total reflection outlet boundary condition at the end of artery 7 , corresponding to a complete occlusion of the vessel (see Fig. 3(b)).

\section{Properties of the network}

Table 1 presents the arterial geometric (length $L$, reference cross-section $A$, thickness $h$ ) and material (Young's modulus $E$ ) properties used in the numerical computations. We adjusted these properties from the literature ([29], [30]) to the chosen 9-artery networks model to correspond to an average human body [52]. The resistance boundary conditions are estimated automatically and reported in the results section in Table 3.

\section{Numerical resolution schemes}

As the system (4) is non-linear, analytical solutions are not available yet. Numerical schemes have been proposed to solve the system ([56], [57]) using finite differences [52], finite volumes [58], finite elements [16], and discontinuous Galerkin methods [59]. The system can be decomposed into a hyperbolic subproblem that accounts for the transport, a parabolic subproblem describing the viscoelastic effect and a reaction subproblem for the friction source term. To solve the hyperbolic subproblem, we chose a finite volume numerical scheme using a kinetic approach [60] with a second-order monotonic upwind scheme for conservation law (MUSCL) reconstruction [61] and a second-order AdamBashforth (AB2) time integration scheme. We used a Cranck-Nicholson scheme for the parabolic subproblem and the same $A B 2$ scheme for the reaction subproblem. We divided the time domain using a constant time step $\Delta t=10^{-4} \mathrm{~s}$ and introduced a spatial mesh to discretize each artery with a constant step $\Delta x=0.4 \mathrm{~cm}$, that are typical values for computation with enough precision. We solved the system using a code developed in our laboratory written in $\mathrm{C}++$. 
(a)

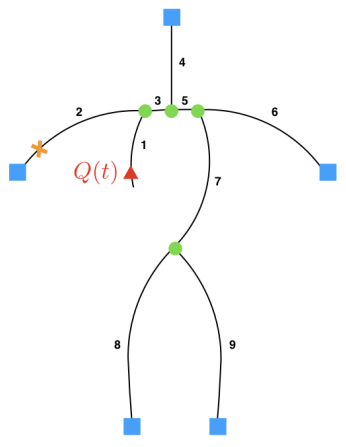

(b)

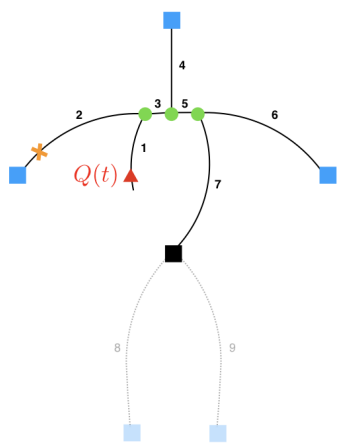

Measuring point

Resistance boundary condition

Bifurcation

FIGURE 3 Schematic representation of the 9-artery network. We represent (a) the configuration without the clamp (pre-clamp), (b) the configuration with the clamp (post-clamp), where we model the occlusion of artery 7 with a total reflection boundary condition. The green circles represent the bifurcations, the light blue squares represent the resistance boundary conditions, the black square is the point of clamping modeled by a reflection coefficient of 1 and the orange cross is the pressure measurement point where we compared experimental and simulated pressure waves.

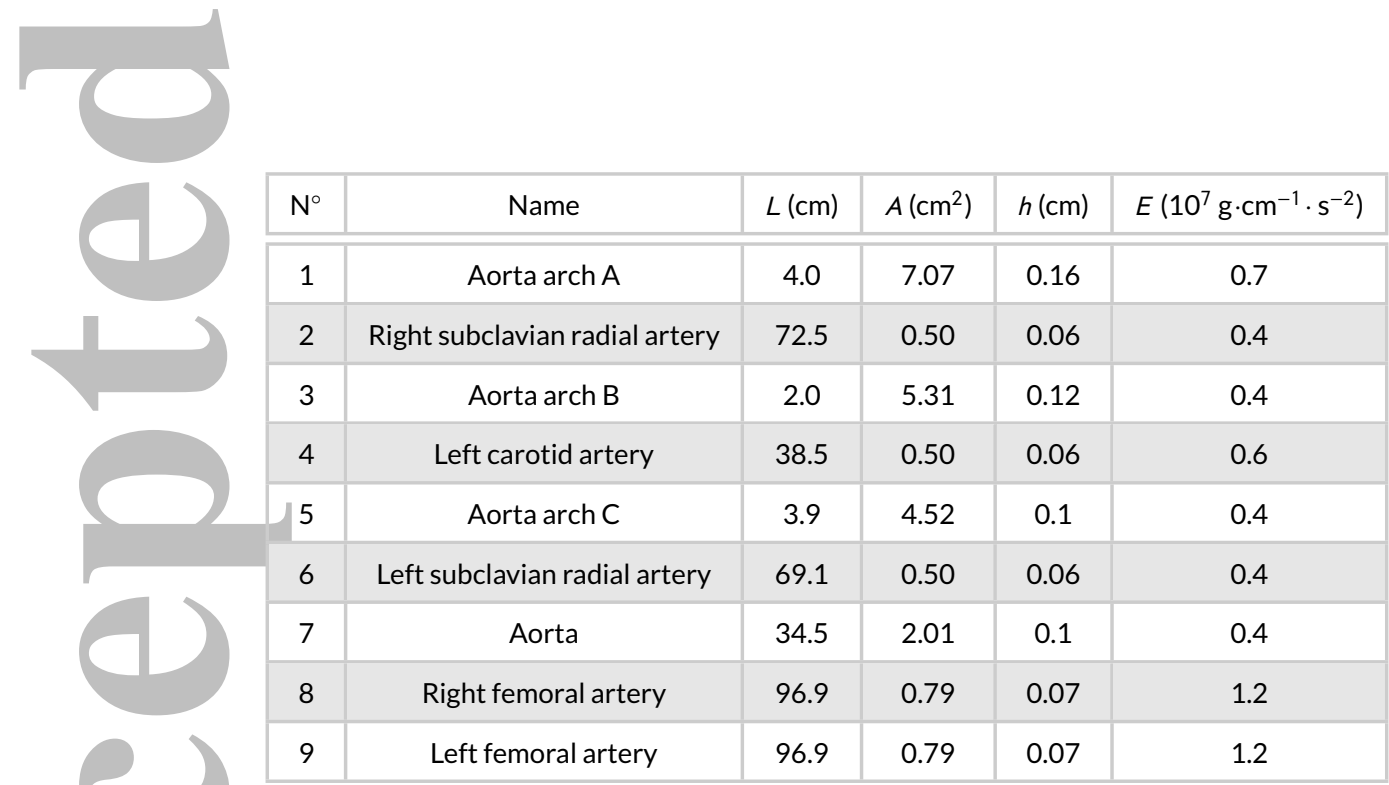

TAB LE 1 Geometric and material properties of the 9-artery network model ([33], [52], [55]) including the length of each vessel $L$, expressed in $\mathrm{cm}$, the reference cross-section $A$ in $\mathrm{cm}^{2}$, the arterial wall thickness $h$ in $\mathrm{cm}$ and the Young's modulus $E$ in $\mathrm{g} \cdot \mathrm{cm}^{-1} \cdot \mathrm{s}^{-2}$, considered constant in each artery. 


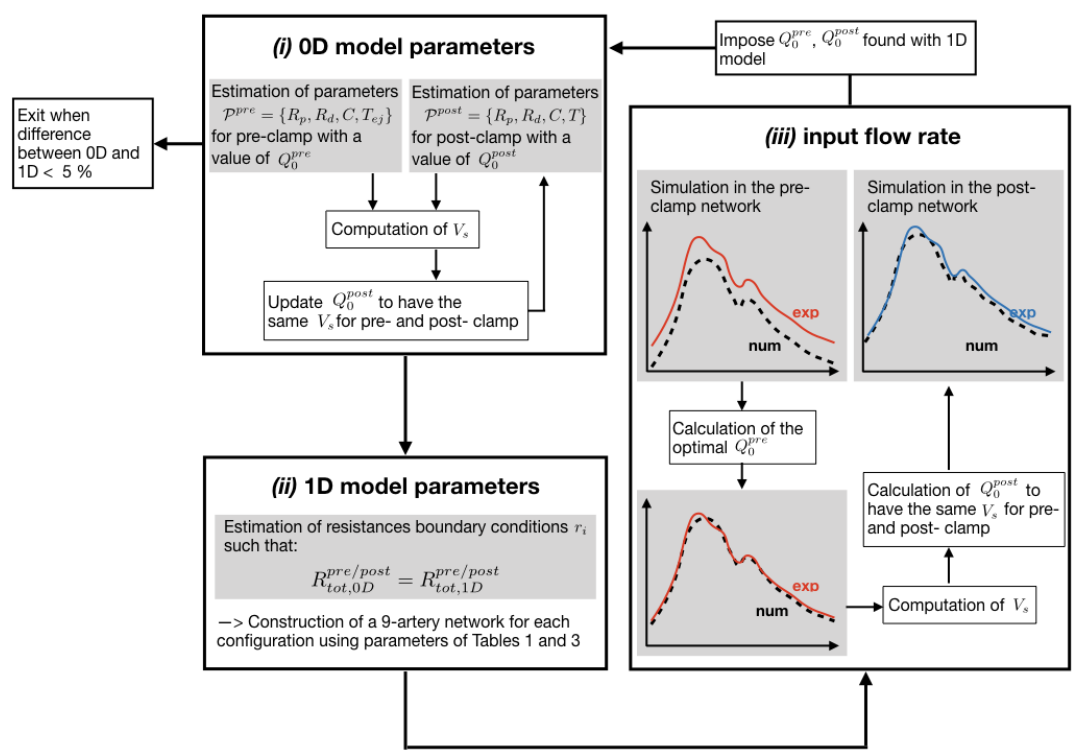

FIGURE 4 Optimization algorithm described in Sec. 2.4 to estimate the patient-specific parameters of the OD and 1D models. The parameters of the OD model are $\mathcal{P}^{p r e}$ for the pre-clamp configuration and $\mathcal{P}^{\text {post }}$ for the post-clamp configuration and are composed of the proximal resistance $R_{p}$, the distal resistance $R_{d}$, the compliance $C$ and the ejection time $T_{e j}$. The flow rate $Q_{0}^{\text {pre }}$ (respectively $Q_{0}^{\text {post }}$ ) corresponds to the amplitude of the input flow rate $Q(t)$ for the pre-clamp configuration (respectively post-clamp). The stroke volume $V_{s}$ is the integral of the flow rate $Q(t)$ over one heart period $T$. The resistances $r_{i}$ are the resistance boundary conditions of the 1D network (see Fig. 5).

\subsection{Parameter estimation}

Typically, during the course of a clinical procedure, little is known about patient-specific vascular properties making parameter estimation a powerful tool to identify these physiological quantities. Thus, we proposed an optimization strategy designed to automatically estimate both $\mathrm{OD}$ and $1 \mathrm{D}$ model parameters using the experimental measurements. Fig. 4 presents the 3-step algorithm of the process in which:

(i) we estimated the OD model parameters: the resistances $\left(R_{p}\right.$ and $\left.R_{d}\right)$, the compliance $(C)$ and the ejection time $\left(T_{e j}\right)$, (ii) we estimated the resistance boundary conditions $r_{i}$ of the $1 \mathrm{D}$ model to match the total resistance of the $1 \mathrm{D}$ model to the total resistance of the $\mathrm{OD}$ model found in step (i),

(iii) we estimated the amplitude of the input flow rate $\left(Q_{0}\right)$ for both models using the $1 \mathrm{D}$ model.

\section{(i) Estimation of the $\mathrm{OD}$ model parameters}

The objective of this first step was to estimate the OD model parameters $\mathcal{P}^{\text {pre }}=\left\{R_{P}^{p r e}, R_{d}^{\text {pre }}, C^{\text {pre }}, T_{e j}^{\text {pre }}\right\}$ for the preclamp and $\mathcal{P}^{\text {post }}=\left\{R_{p}^{\text {post }}, R_{d}^{\text {post }}, C^{\text {post }}, T_{e j}^{\text {post }}\right\}$ for the post-clamp configuration, solving an inverse problem based on the experimental measurements. We defined a cost-function $J$ that characterized the difference between the experimental and simulated pressure waves, respectively $P_{\text {exp }}(t)$ and $P_{\text {num }}(\mathcal{P}, t)$. We minimised this cost-function $J$ with respect to $\mathcal{P}$, the set of parameters, for each configuration. We defined the cost-function as follows: 


$$
J^{\text {pre/post }}(\mathcal{P}, t)=\left(\int_{0}^{T}\left(P_{\exp }(t)-P_{\text {num }}(\mathcal{P}, t)\right)^{2} \mathrm{~d} t\right)^{1 / 2}
$$

where $\mathcal{P}$, the set of parameters $\left\{R_{p}, R_{d}, C, T_{e j}\right\}$, was either $\mathcal{P}^{p r e}$ for the pre-clamp or $\mathcal{P}^{p o s t}$ for the post-clamp configuration.

To calculate $P_{\text {num }}$, we solved Eq. (1) and imposed an arbitrary value of $Q_{0}$ such that the systolic volume $V_{s}$, i.e. ventricular ejection volume over a heartbeat, was within the typical range of 70 to $90 \mathrm{~mL}$. We defined the systolic (or stroke) volume as the integral of the input flow rate $Q(t)$ over one heart period $T$. We imposed the same stroke volume $V_{s}$ in both configurations.

For each configuration we determined the optimal set of parameters $\mathcal{P}$ using the gradient-based method L-BFGS-B (from the initials of the original authors Broyden [62], Fletcher [63], Goldfarb [64], Shanno [65]) using the SciPy library from Python [66]. The L-BFGS-B algorithm is a limited-memory Quasi-Newton method that solves large non-linear optimization problems. It allows bound constraints, in order to maintain each parameter within a physiological range [67]. In particular, we required that all parameters have positive values and that the compliance remains in the interval $C \in\left[10^{-7}, 10^{-1}\right] \mathrm{g}^{-1} \cdot \mathrm{cm}^{4} \cdot \mathrm{s}^{2}$.

The classical gradient method is Newton's iterative method: $\mathcal{P}_{k+1}=\mathcal{P}_{k}-B_{k}^{-1} \nabla J\left(\mathcal{P}_{k}\right)$ where $B_{k}$ is the matrix of the second-order partial derivatives of $J$, called the Hessian matrix and the limit of $\mathcal{P}_{k}$, when the algorithm converges, minimises the function $J$. The BFGS method was designed to avoid constructing the Hessian matrix $B$ and uses instead an approximation of the inverse of the second derivative of $J$ by analyzing the gradient. This approximation allows using quasi-Newton's method to find the minimum in the parameter space. The algorithm is as follows.

We chose an initial guess of the set of parameters $\mathcal{P}_{0}$, initialized the Hessian matrix $B_{0}$ with the identity matrix and repeated the following steps until convergence:

1. find $\mathbf{p}_{\mathrm{k}}$ solving $B_{k} \mathbf{p}_{\mathbf{k}}=-\nabla J\left(\mathcal{P}_{k}\right)$ where $\mathbf{p}_{\mathbf{k}}$ is the direction of the descent and $\mathcal{P}_{k}$ the set of parameters to be estimated at step $k$,

2. find the optimal time step $\alpha_{k}$ in the direction $\mathbf{p}_{\mathbf{k}}$,

3. update the solution: $\mathcal{P}_{k+1}=\mathcal{P}_{k}+\alpha_{k} \mathbf{p}_{\mathbf{k}}=\mathbf{x}_{\mathbf{k}}+\mathbf{s}_{\mathbf{k}}$,

4. calculate $\mathbf{y}_{\mathbf{k}}=\nabla J\left(\mathcal{P}_{k+1}\right)-\nabla J\left(\mathcal{P}_{k}\right)$,

5. update the value of the Hessian matrix using the information from previous iteration:

$$
B_{k+1}=B_{k}+\frac{\mathbf{y}_{\mathbf{k}} \mathbf{y}_{\mathbf{k}}^{T}}{\mathbf{y}_{\mathbf{k}}^{T} \mathbf{s}_{\mathbf{k}}}-\frac{B_{k} \mathbf{s}_{\mathbf{k}} \mathbf{s}_{\mathbf{k}}^{T} B_{k}}{\mathbf{s}_{\mathbf{k}}^{T} B_{k} \mathbf{s}_{\mathbf{k}}} .
$$

Convergence was reached when $\frac{\left(J\left(\mathcal{P}_{k}\right)-J\left(\mathcal{P}_{k+1}\right)\right)}{\max \left(\left|J\left(\mathcal{P}_{k}\right)\right|,\left|J\left(\mathcal{P}_{k+1}\right)\right|, 1\right)} \leq \epsilon$, with $\epsilon=10^{-9}$.

The BFGS method is deterministic, and to ensure that the optimal values are global we explored the stability of the minimum by adding extra noise using the Basin Hopping algorithm [68]. It is a stochastic algorithm that looks for the global minimum of the cost function by creating a random perturbation of the parameters $\mathcal{P}$ at each optimisation step.

This step provided the optimal values of the OD model parameters $\mathcal{P}^{p r e}$ and $\mathcal{P}^{\text {post }}$ for both configurations, in the physiological parameter space, as the global optima of the cost-function $J$.

\section{(ii) Estimation of the resistance boundary conditions of the 1D model}

The objective of this second step was to estimate the resistance boundary conditions $r_{i}$ of the 1D network. We proposed a new parameter estimation problem to identify the resistance boundary conditions $r_{i}$ such that the total resistance of the $1 \mathrm{D}$ model $\tilde{R}_{t o t, 1 D}$ matched that of the OD model $R_{t o t, 0 D}=R_{p}+R_{d}$ found in step (i). Indeed, since the OD total 
(a)

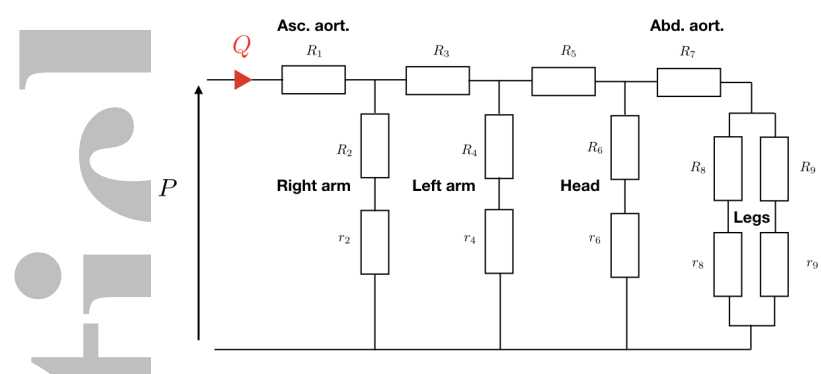

(b)

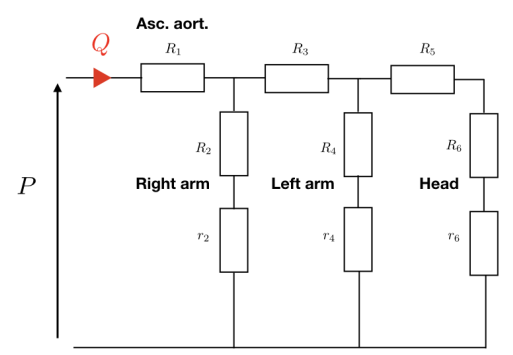

FIGURE 5 Analogy of the 9-artery network with resistors. (a) The pre-clamp network shown in Fig. 3(a) that has a total resistance $\tilde{R}_{\text {tot }, 1 D}^{\text {pre }}$, (b) the post-clamp network from Fig. $3(\mathrm{~b})$ that has a total resistance $\tilde{R}_{\text {tot } 1 D}^{\text {post }}$. The labels of resistors are identical to the labels of arteries from the 1D network (Fig. 3). The Poiseuille (or hydraulic) resistances $R_{i}$, and the resistance boundary conditions $r_{i}$ are reported in Table 3 .

resistance was estimated from real invasive data, it seems reasonable that it is the total resistance of the patient, which explains why we matched the $1 \mathrm{D}$ resistance to the $\mathrm{OD}$ resistance.

We can calculate analytically the total resistance of the $1 \mathrm{D}$ model for both configurations assuming a OD analogy of the 1D network, as represented on Fig. 5. This total resistance $\tilde{R}_{t o t, 1 D}$ depends on the Poiseuille (or hydraulic) resistances $R_{i}$ of each vessel [50]: $R=8 \pi \mu L_{i} / A_{i}^{2}$, where $L_{i}$ is the length of the vessel $i$, and $A_{i}$ the cross-section of the vessel $i$. The values of the Poiseuille resistances $R_{i}$ for each vessel of the 9-artery network are fixed by the geometry (Table 1). The total resistance $\tilde{R}_{t o t, 1 D}$ also depends on the resistance boundary conditions $r_{i}$ of each terminal artery $i$.

We defined a new cost function $J$ in which we included both the pre-clamp and post-clamp values of the total resistance so that the optimal set of $r_{i}$ would preserve the ratio between the pre and post-clamp OD total resistances. As $R_{i} \ll r_{i}$, we only minimized $J$ with respect to the resistance boundary conditions $r_{i}$. The new cost function $J$ is:

$$
J\left(r_{i}\right)=\left(\tilde{R}_{t o t, 1 D}^{p r e}\left(R_{i}, r_{i}\right)-R_{t o t, 0 D}^{p r e}\right)^{2}+\left(\tilde{R}_{t o t, 1 D}^{\text {post }}\left(R_{i}, r_{i}\right)-R_{t o t, 0 D}^{\text {post }}\right)^{2},
$$

where $\tilde{R}_{\text {tot }, 1 D}^{\text {pre }}\left(\right.$ Fig. 5 (a)) and $\tilde{R}_{\text {tot }, 1 D}^{\text {post }}$ (Fig. 5(b)) are the $1 \mathrm{D}$ total resistances for the pre-clamp and post-clamp respectively, $R_{t o t, 0 D}^{\text {pre }}$ and $R_{\text {tot }, O D}^{\text {post }}$ are the $\mathrm{OD}$ total resistances for the pre-clamp and post-clamp respectively.

We used the same Basin-Hopping optimization process to obtain the boundary conditions $r_{i}$ of the 1D network.

(iii) Estimation of the amplitude of the input flow rate for both models

The last parameter we estimated was the amplitude of the input flow rate $Q_{0}$. Indeed, it was not possible to estimate this parameter in step (i). In Eq. (1), when neglecting the compliance, the pressure is linked to the flow rate through $p=R_{\text {tot }} Q$. As we only had pressure data, it was only possible to estimate either the resistance or the flow rate with the OD model. Indeed, the algorithm would always find a balance between these two quantities that would minimise the cost function but that would not necessarily respect physiological values of either of the parameters. The lack of data on the flow rate is a limitation of the present study.

In step (i), we imposed an arbitrary value for the amplitude of the input flow rate $Q_{0}$. In this step, we estimated $Q_{0}$ for both configurations using the 1D model with the constraint of a constant stroke volume, within the range of 70 to $90 \mathrm{~mL}$. We computed the 1D model in the pre-clamp configuration with the input flow rate characterized by the estimated value of the ejection time $T_{e j}$ and the same arbitrary $Q_{0}^{p r e}$ as the OD model. We used a simple gradient-based 
algorithm from the same SciPy library [66] to find the optimal value of the amplitude of the input flow rate $Q_{0}^{\text {pre }}$ for this configuration. Convergence was reached when the correlation between experimental and simulated pressure waves was superior to 0.95 . Then we computed the stroke volume $v_{s}$, calculated the $Q_{0}^{\text {post }}$ so that the stroke volume was identical between both configurations, and finally computed the 1D model for the post-clamp configuration. This last step allowed us to estimate $Q_{0}^{\text {pre }}$ and $Q_{0}^{\text {post }}$ for the two models.

Since $Q_{0}$ changed from step (i) to step (iii), the process was iterated to recalculate the new OD total resistance with the optimal value of $Q_{0}$. We exited the loop when the $O D$ and $1 \mathrm{D}$ models had the same total resistance, the same amplitude of the input flow rate and the same stroke volume between the pre-clamp and post-clamp configurations.

\section{3 | RESULTS}

In this section, we present the results of the OD model (Sec. 3.1) and the 1D model (Sec. 3.2).

\section{$3.1 \mid$ Zero-dimensional results}

Using the $0 \mathrm{D}$ model and the experimental measurements of blood pressure we estimated the patient-specific parameters $\mathcal{P}^{\text {pre }}$ and $\mathcal{P}^{\text {post }}\left(\left\{R_{p}, R_{d}, C, T_{e j}\right\}\right)$ for the pre-clamp and post-clamp configurations respectively. These results, reported in Table 2, showed a 10\% increase in total resistance $R_{\text {tot }}=R_{p}+R_{d}$ and $20 \%$ decrease in compliance $C$ after clamping. Similar changes in the vascular resistance with clamping were found in [10]. Table 2 also showed the changes in heart function, characterized by the three parameters $Q_{0}, T_{e j}$, and $T$. The estimated ejection time $T_{e j}$, that is a percentage of the heart period $T$, did not change significantly. However, the heart period $T$, which was prescribed by the experimental data, increased with clamping. Since we assumed and thus imposed a constant systolic volume, we estimated that the input flow rate $Q_{0}$ decreased by $10 \%$ with clamping.

We present in Fig. 6 a comparison between the experimental and the simulated pressure signal, the latter computed with the optimal sets of parameters $\mathcal{P}^{p r e}$ and $\mathcal{P}^{\text {post }}$. The simulated pressure wave resulted from solving the $0 \mathrm{D}$ equation (1) with the optimal set of parameters obtained with the step (i) of the parameter estimation process. The OD model gave an accurate general description of the pressure curve in both configurations even though the systolic pressure peak was not well reproduced. The correlation coefficients between the simulated and the experimental waves were $\mathrm{R}^{2}=0.95$ for the pre-clamp and $\mathrm{R}^{2}=0.97$ for the post-clamp configuration.

Even though we found a high correlation between the simulated and the experimental pressure signal, we wanted to ensure that the values found with the algorithm minimised the cost function in the physiological parameter space. We therefore evaluated the sensitivity of the cost function $J$ to the parameters of the OD model. We represented in Fig. 7 the cost function $J^{\text {pre }}$ for the pre-clamp configuration as a function of the total resistance $R_{\text {tot }}$ and the compliance $C$ for a fixed ejection time $T_{e j}=31.8 \%$ and a fixed ratio between $R_{p}$ and $R_{d}$ set at 0.165 . Figure 7 showed that the values reported in Table 2 minimised the cost function J. The two black lines on Fig. 7 represent the isovalues of the cost function $J$ that correspond respectively to $a+10 \%$ and $+20 \%$ increase of $J$ with respect to its minimum value. Within the $10 \%$ of $J$, we found a variation of $18 \%$ in $R_{\text {tot }}$ and a variation of $48 \%$ in $C$. This result showed that the cost function $J$ was a lot more convex with respect to $R_{\text {tot }}$ than $C$, meaning that the model was more sensitive to a change in total resistance than in compliance. 


\section{(1)}

\begin{tabular}{|c|c|c|c|c|c|c|c|}
\hline & \multicolumn{3}{|c|}{ Model parameters } & \multicolumn{4}{|c|}{ Heart function } \\
\hline & $R_{p}$ & $R_{d}$ & C & $T_{e j}$ & $T$ & $Q_{0}$ & $V_{s}$ \\
\hline Pre-clamp & 205 & 1240 & $1.29 e-3$ & 31.8 & 1.15 & 375 & 87.2 \\
\hline Post-clamp & 260 & 1313 & $1.04 \mathrm{e}-3$ & 32.1 & 1.283 & 340 & 89 \\
\hline Changes between pre/post & $+27 \%$ & $+6 \%$ & $-20 \%$ & $+1 \%$ & $+12 \%$ & $-10 \%$ & - \\
\hline
\end{tabular}

TAB LE 2 Summary of parameter estimation results using the Windkessel model. Resistances $R_{p}$ and $R_{d}$ are expressed in dyn $\cdot \mathrm{cm}^{-5} \cdot \mathrm{s}$, the compliance $C$ is in $\mathrm{g}^{-1} \cdot \mathrm{cm}^{4} \cdot \mathrm{s}^{2}$, the ejection time $T_{e j}$ is expressed as a percentage of the heart period, $T$ the heart period is in s, the amplitude of flow rate $Q_{0}$ is in $\mathrm{cm}^{3} / \mathrm{s}$ and the stroke volume $V_{s}$ is in $\mathrm{cm}^{3}$.
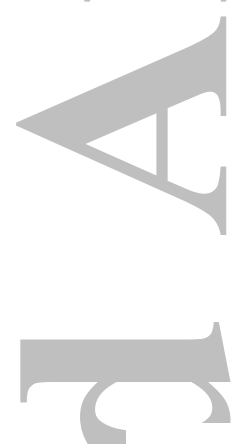

(a)

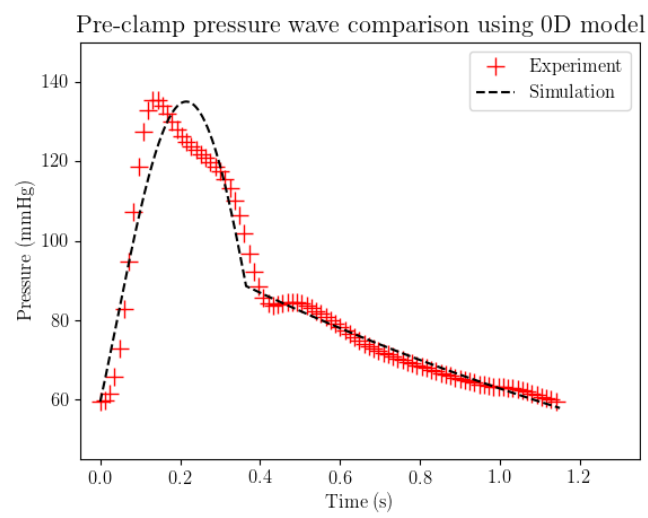

(b)

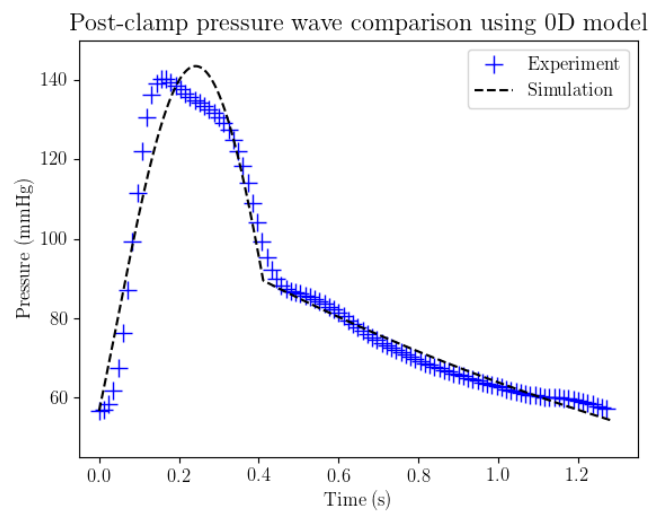

FIGURE 6 Comparison between experimental and numerical pressure signals using the OD model for a patient undergoing aortic clamping during vascular surgery. (a) Pre-clamp configuration, (b) post-clamp configuration. Colored + symbols are experimental pressure waves, black dashed lines (- - -) are simulated pressure waves solving Eq. (3). For the pre-clamp situation, the correlation coefficient between the experimental and simulated curve was $R^{2}=0.95$ and for the post-clamp situation $\mathrm{R}^{2}=0.97$. 


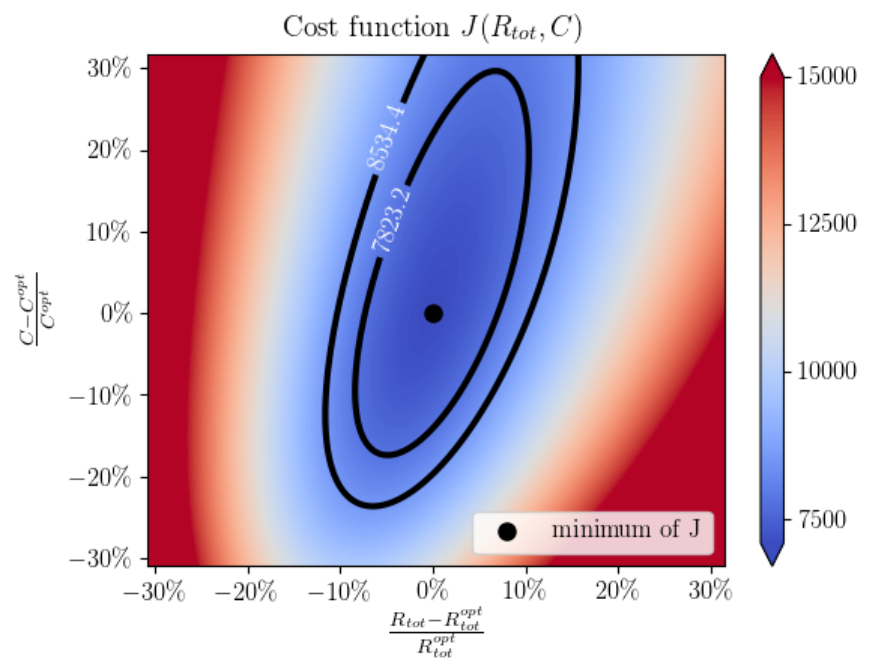

FIGURE 7 Contour plot of the cost function $J$ for Fig. 6(a) as a function of the model parameters $R_{\text {tot }}$ and $C$ expressed as percentage of the optimal values $R_{\text {tot }}^{o p t}$ and $C^{o p t}$ respectively (values reported in Table 2), with a fixed ratio between $R_{d}$ and $R_{p}$ of 0.165 and a fixed ejection time $T_{e j}=31.8 \%$ for the pre-clamp situation. The black dot represents the minimum of $J$ estimated with the optimization process described in Sec. 2.4. Black solid lines $(-)$ represent the isovalues of $J$ at $a+10$ and $+20 \%$ increase of the minimum of the cost function $J$.

\subsection{One-dimensional results}

The algorithm presented in Sec. 2.4 allowed us to estimate the values of the resistance boundary conditions $r_{i}$, reported in Table 3. The values of $r_{i}$ corresponded to physiological values of the peripheral resistances and were similar to those reported in the literature [35]. As described in Sec. 2.4, the resistance boundary conditions $r_{i}$ of the 1D networks were estimated such that the total resistance matched that of the OD model. For this reason, there was the same $10 \%$ increase in total resistance as the OD model between the pre-clamp and post-clamp configurations with the 1D model. We observed that both the pre- and post-clamp configurations were fitted without any change in the resistance boundary conditions which means that there was no change in peripheral resistance with clamping. As we imposed a constant stroke volume, the post-clamp configuration was fitted with a $10 \%$ decrease of the amplitude of the input flow rate compared to the pre-clamp configuration (see Table 2).

Similarly to the OD model, we compared the experimental and simulated pressure curves on Fig. 8. The simulated pressure wave resulted from solving the 1D equations (4) in the 9-artery network with the optimal resistance boundary conditions $r_{i}$ obtained with the step (ii) of the parameter estimation process, presented in Sec. 2.4. The correlation coefficients between the simulated and the experimental pressure waves were $R^{2}=0.96$ for the pre-clamp and $R^{2}=0.97$ for the post-clamp configuration. 
(a)

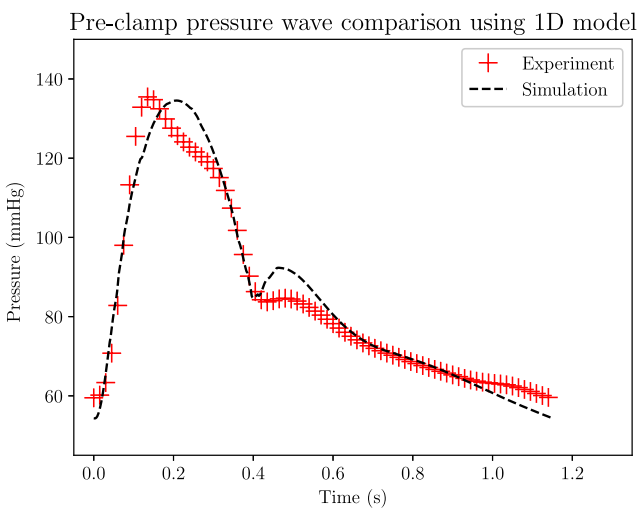

(b)

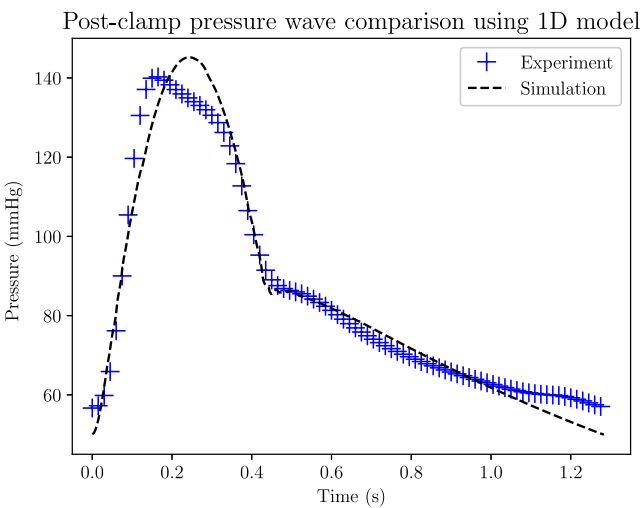

FIGURE 8 Comparison between experimental and numerical pressure waves using the 1D model for a patient undergoing aortic clamping during vascular surgery. (a) Pre-clamp configuration, (b) post-clamp configuration. Colored + symbols are experimental pressure waves, black dashed lines (- - -) are simulated pressure waves solving Eq. (4). For the pre-clamp situation, the correlation coefficient between the experimental and simulated curve was $R^{2}=0.96$ and for the post-clamp situation $\mathrm{R}^{2}=0.97$.

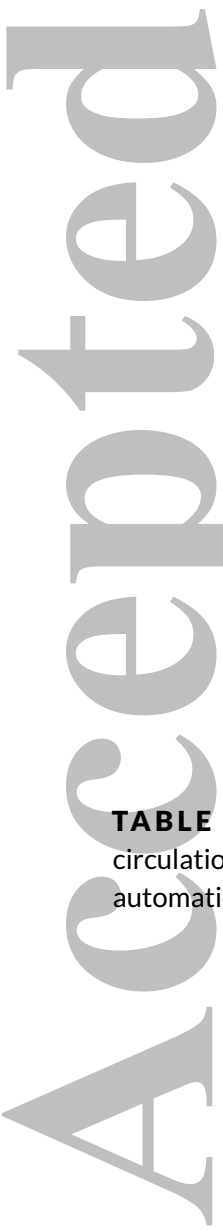

\begin{tabular}{|c|c|c|}
\hline $\mathrm{N}^{\circ}$ & Name & $r_{i}\left(10^{3} \mathrm{dyn} \cdot \mathrm{cm}^{-5} \cdot \mathrm{s}\right)$ \\
\hline 1 & Aorta arch A & - \\
\hline 2 & Right subclavian radial artery & 4.6 \\
\hline 3 & Aorta arch B & - \\
\hline 4 & Left carotid artery & 4.7 \\
\hline 5 & Aorta arch C & - \\
\hline 6 & Left subclavian radial artery & 4.6 \\
\hline 7 & Aorta & - \\
\hline 8 & Right femoral artery & 30.3 \\
\hline 9 & Left femoral artery & 30.3 \\
\hline
\end{tabular}

TA B LE 3 Resistance boundary conditions $r_{i}$, expressed in dyn $\cdot \mathrm{cm}^{-5} \cdot \mathrm{s}$, of each segment modeling the peripheral circulation in the capillaries, imposed at the termination of the 1D 9-artery networks. The values of $r_{i}$ were estimated automatically using the results from the OD model with the 3-step process presented in Sec. 2.4. 


\section{DISCUSSION}

We have shown that the OD model gives an accurate description of the pressure waves in both the pre-clamp and post-clamp configurations (Fig. 6) and offers information on relevant global vascular parameters. Even though lumped analysis may initially be considered outdated, these results indicate that a simple and computationally efficient inverse method is enough to provide access to patient-specific vascular properties. Nevertheless, an important limitation of the OD model is that it does not take into consideration wave propagation and reflection. These are currently considered major phenomena in vascular physiology and pathophysiology, since arterial stiffness studied through Pulse Wave Velocity (PWV) has shown to have a crucial role in the pathogenesis of arterial hypertension and atherosclerosis [43]. In particular, the $\mathrm{OD}$ model has important limitations for describing the pressure wave during diastole, since it only fits the diastolic regime with an exponential function.

One of the main results of this paper is the $10 \%$ increase in total resistance and $20 \%$ decrease in total compliance after aortic clamping. These results can be interpreted by considering that the OD model sees the arterial network as an assembly of resistive and compliant vessels connected in parallel. Since the total resistance of a network is the inverse of the sum of the inverse of each vessel resistance, when removing vessels from the circuit (i.e., when clamping), the total resistance should increase. This increase in resistance is in accordance with previous experimental findings ([10], [11], [13]) that have described hemodynamics changes 5 to 30 minutes after clamping using invasive methods such as transesophageal echocardiography or pulmonary artery catheters. Similarly, as the total compliance is the sum of all vessel compliances, the total compliance should decrease with clamping. Though these results may be qualitatively anticipated following this reasoning, the quantification of these changes is only possible through parameter estimation using the Windkessel model. In addition, the study of the hemodynamic impact of aortic clamping has usually focused on systemic arterial pressure and vascular resistance, but vascular compliance has not been reported since clinical attention has been drawn on this parameter only recently [43].

A sensitivity analysis of the $\mathrm{OD}$ model showed that the model is much more sensitive to changes in total resistance than in compliance. This would suggest that the algorithm estimation of the total resistance of the patient has a high level of precision, while there is more uncertainty surrounding the estimation of the compliance of the patient. Nonetheless, given that most methods in the literature that estimate arterial compliance are based on two- or three-element Windkessel models [69], it is reasonable to assume that this is currently the state of the art for a minimally-invasive estimation of vascular compliance.

Similarly to the OD model, the 1D model gives an accurate description of the pressure waves in both the pre-clamp and post-clamp configurations (Fig. 8). However, as opposed to the OD model, the 1D model accurately reproduced the diastolic part of the experimental pressure waves in both the pre-clamp and post-clamp configurations. In particular, the 1D model captured the dicrotic notch [41], a small and brief increase in arterial pressure when the aortic valve closes, as the model accounts for the propagation and reflection of the pulse waves. The 1D model, as an assembly of tubes in which we solved the fluid equations with only a few weak hypotheses, allowed recovering the physical properties of the arterial pressure wave that were not considered with the OD model. We can thus consider that the 1D model is an improvement of the $\mathrm{OD}$ model and allows its validation by giving similar results.

The fitting of the pressure waves with the 1D model was achieved for both configurations without changing the resistance boundary conditions. It suggests that numerical clamping, i.e. imposing a total reflection at the end of the abdominal aorta, by itself can reproduce and even predict the impact of surgical clamping on the pressure wave morphology. This result was observed under the assumption that the peripheral resistances would not be affected by clamping because of the short acquisition time. We believe the hypothesis of constant peripheral resistances after clamping would no longer be valid in the long-term due to neurohormonal reactions that occur during longer time scales. 
In conclusion, determining the patient-specific total resistance along with the distribution of peripheral resistances with the parameter estimation process is sufficient for the 1D model to accurately reproduce the impact of clamping on the pressure waveform.

One drawback of this study is that we used a very reduced arterial network, composed of only nine arteries for the 1D modeling. Though limited in size, we believe that the network contains the minimal number of arteries needed to describe an average human body. Larger networks (like in [44]) increase the costs, the complexity of the problem and require more sophisticated methods to estimate the resistance boundary conditions, as there are more terminal vessels. In our case, the values from the OD model were a good starting point for the 9-artery model. Future work will focus on developing an efficient identification technique for a large number of parameters of the 1D model which would constitute a major improvement of this line of research.

The main drawback of the method is that we do not have access to experimental information on continuous heart flow rate. This variable can be calculated in critically ill patients with an invasive central vein catheter using the thermodilution method or during cardiovascular surgeries in high-risk patients with transesophageal echocardiography using continuous-wave Doppler assessment. In this study, neither method was available. Hence, the peak systolic pressure cannot be described in either model because the shape of the model input heart signal (Fig. 2(a)) is simplified compared to the physiological heart signal. Moreover, the amplitude of the input flow rate $Q_{0}$ cannot be estimated automatically with the $\mathrm{OD}$ model because its value is linked to pressure through the total resistance that we estimated. Nonetheless, we showed that the models can assess the change in $Q_{0}$ with clamping when assuming that the stroke volume is constant. This change in the amplitude of the input flow rate might be a consequence of our assumption of a constant stroke volume. Due to the lack of experimental comparative data, we cannot verify this assumption, which is a limitation of our study. Despite this limitation, we feel that a constant systolic volume is a safe assumption to make for at least three reasons. First, there is no clear prior evidence in the literature that suggests otherwise. Experimental data on changes in systolic volume during clamping are available from several small-sized clinical observational studies in adult patients undergoing cardiovascular surgeries. However, some data suggest that systolic volume decreases ([12], [9], [7]), while others suggest that it stays the same ([13], [11], [3]). These inconsistent findings are possibly due to differences in loading conditions, surgery techniques, and patient comorbidities [3]. A second reason to support our assumption of a constant systolic volume is that, since we are interested in studying only the immediate impact of clamping, changes in systolic volume during this short time frame, if any, could be considered as non-significant. Finally, if there were any significant changes due to clamping during this short time frame, they would involve a decrease, not an increase, in systolic volume. However, since our patients all had a preserved left ventricular ejection fraction (i.e. a healthy heart), the systolic function of the left ventricle, and therefore the stroke volume, is not expected to decrease during clamping. For these three reasons, we believe that, in spite of the lack of experimental data, a constant systolic volume is a safe assumption to make.

Overall, this study offers evidence supporting the use of numerical models to assess physiological quantities that cannot be easily measured by physicians (vascular compliance) or that can only be measured invasively by a central vein catheter (vascular resistance) under a steady-state. This information on immediate changes in the vascular parameters could be useful for intraoperative patient monitoring and for assessing vascular prosthesis safety, thereby adding valuable information for decision-making in critical care scenarios. Considering that the postoperative mortality rate for elective AA repair surgery is one of the highest, we believe it is crucial to understand the effects of clamping to minimize the risks of complication. 


\section{CONCLUSION}

The objective of this work was to investigate the immediate impact of clamping during vascular surgery, as it has not previously been explored with macroscopic blood flow models. We compared experimental invasive measurements of pressure in the right radial artery for a patient undergoing abdominal aneurysm repair surgery with two numerical approaches (OD and 1D). Both approaches gave a different level of knowledge on the changes that occur immediately after clamping. We developed an inverse method based on experimental data to identify patient-specific parameters using a three-element Windkessel OD model for the systemic circulation. We highlighted two main changes in the vascular properties of the systemic circulation after clamping: on one hand, total resistance increased by $10 \%$, and on the other hand, the total compliance decreased by $20 \%$. In this work, we reported the values of the model parameters obtained for a patient undergoing AA repair surgery. We showed that these values minimised the difference between the experimental pressure wave and simulated pressure wave. This model allowed to determine resistance and compliance, which are useful parameters for interpreting a patient's hemodynamic condition in a critical care scenario, and which cannot be measured non-invasively. Then we used a 1D model and developed a second parameter estimation technique to identify the values of the resistance boundary conditions. We observed that the numerical clamping can predict and reproduce the morphology of the experimental curve without changes in peripheral resistances. On the contrary, heart function adjusted slightly under the action of clamping. We assumed that the systolic volume would not change before and after the clamping event because the data acquisition was carried out in a very short time range. This assumption led to a $10 \%$ decrease in the amplitude of the input flow rate during clamping due to an increase in the heart period. Any automatic parameter identification method for problems with a large number of parameters would represent an improvement to the present work.

\section{ACKNOWLEDGEMENTS}

We would like to thank the anesthesiologists, in particular Ismail Khelifa, and nurses from the vascular surgery ward at the Hôpital Universitaire Pitié-Salpêtrière for their help and support in this study. We acknowledge ECOS-Sud 2016, Project Number: A16S03 and PICT 2015-2168 grant.

\section{CONFLICT OF INTEREST}

None declared.

\section{ETHICAL REVIEW}

The study is in accordance with the ethical principles of the Declaration of Helsinki [70]. The study protocol was approved by the institutional review board of the Hôpitaux Universitaires La Pitié-Salpêtrière. Since this was an observational study involving only routine clinical practices and therefore offering minimal risk for patients, the need for an informed consent form was waived.

\section{REFERENCES}

[1] MEMBERS WG, Benjamin EJ, Blaha MJ, Chiuve SE, Cushman M, Das SR, et al. Heart disease and stroke statistics-2017 update: a report from the American Heart Association. Circulation 2017;135(10):e146. 
[2] Erbel R, Aboyans V, Boileau C, Bossone E, Di Bartolomeo R, Eggebrecht H, et al. 2014 ESC Guidelines on the Diagnosis and Treatment of Aortic Diseases. Revista Espanola de Cardiologia (English Edition) 2015;3(68):242.

[3] Zammert M, Gelman S. The pathophysiology of aortic cross-clamping. Best Practice \& Research Clinical Anaesthesiology 2016;30(3):257-269.

[4] Politi MT, Wray SA, Fernández JM, Gaudric J, Ghigo AR, Lagrée PY, et al. Impact of arterial cross-clamping during vascular surgery on arterial stiffness measured by the augmentation index and fractal dimension of arterial pressure. Health and technology 2016;6(3):229-237.

[5] Salsano A, Giacobbe DR, Sportelli E, Olivieri GM, Natali R, Prevosto M, et al. Aortic cross-clamp time and cardiopulmonary bypass time: prognostic implications in patients operated on for infective endocarditis. Interactive cardiovascular and thoracic surgery 2018;27(3):328-335.

[6] Ruggieri VG, Bounader K, Verhoye JP, Onorati F, Rubino AS, Gatti G, et al. Prognostic impact of prolonged cross-clamp time in coronary artery bypass grafting. Heart, Lung and Circulation 2018;27(12):1476-1482.

[7] Biais M, Calderon J, Pernot M, Barandon L, Couffinhal T, Ouattara A, et al. Predicting fluid responsiveness during infrarenal aortic cross-clamping in pigs. Journal of cardiothoracic and vascular anesthesia 2013;27(6):1101-1107.

[8] Li C, Li YS, Xu M, Wen SH, Yao X, Wu Y, et al. Limb Remote Ischemic Preconditioning for Intestinal and Pulmonary Protection during Elective Open Infrarenal Abdominal Aortic Aneurysm RepairA Randomized Controlled Trial. The Journal of the American Society of Anesthesiologists 2013;118(4):842-852.

[9] Kotake Y, Yamada T, Nagata H, Takeda J, Shimizu H. Descending aortic blood flow during aortic cross-clamp indicates postoperative splanchnic perfusion and gastrointestinal function in patients undergoing aortic reconstruction. British journal of anaesthesia 2012;108(6):936-942.

[10] Klotz KF, Klingsiek S, Singer M, Wenk H, Eleftheriadis S, Kuppe H, et al. Continuous measurement of cardiac output during aortic cross-clamping by the oesophageal Doppler monitor ODM 1. BJA: British Journal of Anaesthesia 1995;74(6):655-660.

[11] Montenij LJ, Buhre WF, De Jong SA, Harms JH, Van Herwaarden JA, Kruitwagen CL, et al. Arterial pressure waveform analysis versus thermodilution cardiac output measurement during open abdominal aortic aneurysm repair: A prospective observational study. European Journal of Anaesthesiology (EJA) 2015;32(1):13-19.

[12] Lafanechère A, Albaladejo $P$, Raux $M$, Geeraerts $T$, Bocquet R, Wernet $A$, et al. Cardiac output measurement during infrarenal aortic surgery: echo-esophageal Doppler versus thermodilution catheter. Journal of cardiothoracic and vascular anesthesia 2006;20(1):26-30.

[13] Martín-Cancho MF, Crisóstomo V, Soria F, Calles C, Sánchez-Margallo FM, Díaz-Güemes I, et al. Physiologic responses to infrarenal aortic cross-clamping during laparoscopic or conventional vascular surgery in experimental animal model: comparative study. Anesthesiology research and practice 2008;2008.

[14] Famaey N, Sommer G, Vander Sloten J, Holzapfel GA. Arterial clamping: finite element simulation and in vivo validation. Journal of the mechanical behavior of biomedical materials 2012;12:107-118.

[15] Fereidoonnezhad B, Naghdabadi R, Holzapfel G. Stress softening and permanent deformation in human aortas: continuum and computational modeling with application to arterial clamping. Journal of the mechanical behavior of biomedical materials 2016;61:600-616.

[16] Kim H, Vignon-Clementel I, Coogan J, Figueroa C, Jansen K, Taylor C. Patient-specific modeling of blood flow and pressure in human coronary arteries. Annals of biomedical engineering 2010;38(10):3195-3209.

[17] Vignon-Clementel IE, Marsden AL, Feinstein JA. A primer on computational simulation in congenital heart disease for the clinician. Progress in Pediatric Cardiology 2010;30(1-2):3-13. 
[18] Xiao N, Humphrey JD, Figueroa CA. Multi-scale computational model of three-dimensional hemodynamics within a deformable full-body arterial network. Journal of computational physics 2013;244:22-40.

[19] Westerhof N, Lankhaar JW, Westerhof BE. The arterial Windkessel. Medical \& Biological Engineering \& Computing 2009 Feb;47(2):131-141. https://doi .org/10.1007/s11517-008-0359-2.

[20] Frank O. Die Grundform des Arteriellen Puls. Z Biof 1899;37:483-526.

[21] Stergiopulos N, Westerhof BE, Westerhof N. Total arterial inertance as the fourth element of the windkessel model. American Journal of Physiology-Heart and Circulatory Physiology 1999;276(1):H81-H88.

[22] Milišić V, Quarteroni A. Analysis of lumped parameter models for blood flow simulations and their relation with 1D models. ESAIM: Mathematical Modelling and Numerical Analysis 2004;38(4):613-632.

[23] Muller LO, Toro EF. A global multiscale mathematical model for the human circulation with emphasis on the venous system. International Journal for Numerical Methods in Biomedical Engineering 2014;30:681-725.

[24] Formaggia L, Lamponi D, Tuveri M, Veneziani A. Numerical modeling of $1 D$ arterial networks coupled with a lumped parameters description of the heart. Computer Methods in Biomechanics and Biomedical Engineering 2006 Oct;9(5):273288.

[25] Mynard J, Davidson M, Penny D, Smolich J. A simple, versatile valve model for use in lumped parameter and onedimensional cardiovascular models. International Journal for Numerical Methods in Biomedical Engineering 2012;28(67):626-641.

[26] Quarteroni A, Veneziani A, Vergara C. Geometric multiscale modeling of the cardiovascular system, between theory and practice. Computer Methods in Applied Mechanics and Engineering 2016;302:193-252.

[27] Audebert C, Bekheit M, Bucur P, Vibert E, Vignon-Clementel IE. Partial hepatectomy hemodynamics changes: Experimental data explained by closed-loop lumped modeling. Journal of Biomechanics 2017 Jan;50:202-208.

[28] Ryu J, Hu X, Shadden SC. A coupled lumped-parameter and distributed network model for cerebral pulse-wave hemodynamics. Journal of biomechanical engineering 2015;137(10):101009.

[29] Avolio AP. Multi-branched model of the human arterial system. Medical and Biological Engineering and Computing 1980;18(6):709-718.

[30] Westerhof N, Bosman F, De Vries CJ, Noordergraaf A. Analog studies of the human systemic arterial tree. Journal of biomechanics 1969;2(2):135-136.

[31] Vignon-Clementel IE, Figueroa C, Jansen K, Taylor C. Outflow boundary conditions for 3D simulations of non-periodic blood flow and pressure fields in deformable arteries. Computer methods in biomechanics and biomedical engineering 2010;13(5):625-640.

[32] Moghadam ME, Vignon-Clementel IE, Figliola R, Marsden AL, Investigators MOCHAM, et al. A modular numerical method for implicit OD/3D coupling in cardiovascular finite element simulations. Journal of Computational Physics 2013;244:63-79.

[33] Ghigo AR, Abou Taam S, Wang XF, Lagrée PY, Fullana JM. One-dimensional Arterial Network Model for Bypass Grafts Assessment. Medical Engineering and Physics 2016;43:39-47.

[34] Guan D, Liang F, Gremaud PA. Comparison of the Windkessel model and structured-tree model applied to prescribe outflow boundary conditions for a one-dimensional arterial tree model. Journal of biomechanics 2016;49(9):1583-1592.

[35] Alastruey J, Khir AW, Matthys KS, Segers P, Sherwin SJ, Verdonck PR, et al. Pulse wave propagation in a model human arterial network: Assessment of 1-D visco-elastic simulations against in vitro measurements. Journal of biomechanics 2011;44:2250-2258. 
[36] Nichols WW, O'Rourke MF, C V. McDonald's Blood Flow in Arteries: Theoretical, Experimental and Clinical principles. Hodder Arnold; 2011.

[37] Euler L. Principia pro motu sanguinis per arterias determinando. Opera posthuma mathematica et physica anno 1844 detecta. $1775 ; 2: 814-823$.

[38] Van de Vosse FN, Stergiopulos N. Pulse Wave Propagation in the Arterial Tree. Annual Review of Fluid Mechanics 2011;43:467-499.

[39] Alastruey J, Parker KH, Sherwin SJ. Arterial pulse wave hemodynamics. 11th International Conference on Pressure Surges 2012;30:401-443.

[40] Sherwin SJ, Formaggia L, Peiró J, Franke V. Computational modelling of 1D blood flow with variable mechanical properties and its application to the simulation of wave propagation in the human arterial system. International Journal for Numerical Methods in Fluids 2003 Oct;43(6-7):673-700.

[41] Politi MT, Ghigo A, Fernández JM, Khelifa I, Gaudric J, Fullana JM, et al. The dicrotic notch analyzed by a numerical model. Computers in biology and medicine 2016;72:54-64.

[42] Ghigo AR, Wang XF, Armentano R, Fullana JM, Lagrée PY. Linear and Nonlinear Viscoelastic Arterial Wall Models: Application on Animals. Journal of Biomechanical Engineering 2017 Jan;139(1):011003.

[43] Williams B, Mancia G, Spiering W, Agabiti Rosei E, Azizi M, Burnier M, et al. 2018 ESC/ESH Guidelines for the management of arterial hypertension. European heart journal 2018;39(33):3021-3104.

[44] Lombardi D. Inverse problems in 1D hemodynamics on systemic networks: A sequential approach. International journal for numerical methods in biomedical engineering 2014;30(2):160-179.

[45] Lal R, Mohammadi B, Nicoud F. Data assimilation for identification of cardiovascular network characteristics. International journal for numerical methods in biomedical engineering 2017;33(5):e2824.

[46] Alastruey J, Parker KH, Peiró J, Sherwin SJ. Lumped parameter outflow models for 1-D blood flow simulations: effect on pulse waves and parameter estimation. Communications in Computational Physics 2008;4(2):317-336.

[47] Lagrée PY. An inverse technique to deduce the elasticity of a large artery. The European Physical Journal-Applied Physics 2000;9(2):153-163.

[48] Ismail M, Wall WA, Gee MW. Adjoint-based inverse analysis of windkessel parameters for patient-specific vascular models. Journal of Computational Physics 2013;244:113-130.

[49] Spilker RL, Taylor CA. Tuning multidomain hemodynamic simulations to match physiological measurements. Annals of biomedical engineering 2010;38(8):2635-2648.

[50] Formaggia L, Quarteroni A, Veneziani A. Cardiovascular Mathematics: Modeling and simulation of the circulatory system, vol. 1. Springer Science \& Business Media; 2010.

[51] Smith NP, Pullan AJ, Hunter PJ. An Anatomically Based Model of Transient Coronary Blood Flow in the Heart. SIAM Journal on Applied Mathematics 2001;62(3):990-1018. http: //www . jstor . org/stable/3061797.

[52] Saito M, Ikenaga Y, Matsukawa M, Watanabe Y, Asada T, Lagrée PY. One-Dimensional Model for Propagation of a Pressure Wave in a Model of the Human Arterial Network: Comparison of Theoretical and Experimental Results. Journal of Biomechanical Engineering 2011;133.

[53] Stettler JC, Niederer P, Anliker M. Theoretical analysis of arterial hemodynamics including the influence of bifurcations. Annals of biomedical engineering 1981;9(2):145-164. 
[54] Mynard JP, Valen-Sendstad K. A unified method for estimating pressure losses at vascular junctions. International journal for numerical methods in biomedical engineering 2015;31(7).

[55] Wang JJ, Parker KH. Wave propagation in a model of the arterial circulation. Journal of biomechanics 2004;37(4):457470.

[56] Wang X, Fullana JM, Lagrée PY. Verification and comparison of four numerical schemes for a 1D viscoelastic blood flow model. Computer methods in biomechanics and biomedical engineering 2015;18(15):1704-1725.

[57] Boileau E, Nithiarasu P, Blanco PJ, Müller LO, Fossan FE, Hellevik LR, et al. A benchmark study of numerical schemes for one-dimensional arterial blood flow modelling. International Journal for Numerical Methods in Biomedical Engineering 2015 Jul;31(10):n/a-n/a.

[58] Delestre O, Lagrée PY. A 'well-balanced'finite volume scheme for blood flow simulation. International Journal for Numerical Methods in Fluids 2013;72(2):177-205.

[59] Mynard JP, Nithiarasu P. A 1D arterial blood flow model incorporating ventricular pressure, aortic valve and regional coronary flow using the locally conservative Galerkin (LCG) method. Communications in Numerical Methods in Engineering 2008;24(5):367-417.

[60] Audebert C, Bucur P, Bekheit M, Vibert E, Vignon-Clementel IE, Gerbeau JF. Kinetic scheme for arterial and venous blood flow, and application to partial hepatectomy modeling. Computer Methods in Applied Mechanics and Engineering 2017;314:102-125.

[61] Ghigo AR, Delestre O, Fullana JM, Lagrée PY. Low-Shapiro hydrostatic reconstruction technique for blood flow simulation in large arteries with varying geometrical and mechanical properties. Journal of Computational Physics 2017;331:108-136.

[62] Broyden CG. Quasi-Newton methods and their application to function minimisation. Mathematics of Computation 1967;21(99):368-381.

[63] Fletcher R, Powell MJD. A rapidly convergent descent method for minimization. The computer journal 1963;6(2):163168.

[64] Goldfarb D. A family of variable-metric methods derived by variational means. Mathematics of computation 1970;24(109):23-26.

[65] Shanno DF. Conditioning of Quasi-Newton Methods for Function Minimization. Mathematics of Computation 1970;24(111):647-656.

[66] Jones E, Oliphant T, Peterson P. \{SciPy $\}$ : Open source scientific tools for $\{$ Python $\} 2014 ;$.

[67] Byrd RH, Lu P, Nocedal J, Zhu C. A Limited Memory Algorithm for Bound Constrained Optimization. SIAM Journal on Scientific Computing 1995;16(5):1190-1208. https://doi .org/10.1137/0916069.

[68] Wales DJ, K DJP. Global Optimization by Basin-Hopping and the Lowest Energy Structures of Lennard-Jones Clusters Containing up to 110 Atoms. Journal of Physical Chemistry A 1997;101:5111-5116.

[69] Stergiopulos N, Meister J, Westerhof N. Evaluation of methods for estimation of total arterial compliance. American Journal of Physiology-Heart and Circulatory Physiology 1995;268(4):H1540-H1548.

[70] Association WM, et al. World Medical Association Declaration of Helsinki. Ethical principles for medical research involving human subjects. Bulletin of the World Health Organization 2001;79(4):373. 
